\title{
Oxidative Stress in Type 2 Diabetes Mellitus - A Key Factor for Major Complications
}

\author{
Darshana Kottahachchi ${ }^{\mathrm{a}}$, Erandie Perera $^{\mathrm{b}}$, Ridmi Maddumage ${ }^{\mathrm{b}}$, Mohammad \\ Azam Mansoor ${ }^{\mathrm{C}}$ \\ adarsha.uda@gmail.com \\ ${ }^{a}$ Department of Medical Laboratory Sciences, Faculty of Allied Health Sciences, General Sir John Kotelawala Defence University, \\ Ratmalana (10380), Sri Lanka. \\ ${ }^{b}$ Department of Basic Sciences, Faculty of Allied Health Sciences, General Sir John Kotelawala Defence University, Ratmalana (10380), \\ Sri Lanka. \\ ${ }^{c}$ Department of Natural Sciences, Faculty of Engineering and science, University of Agder, Kristiansand (4611), Norway.
}

\begin{abstract}
Diabetic Mellitus (DM) is considered as a metabolic disorder resulting from flaws in the secretion of insulin, action of insulin or both together. The international diabetes federation reported that approximately 5.0 million deaths were assigned to diabetes mellitus in 2017 and it accounted for $9.9 \%$ of all causes of mortality among people globally. It has been reported that 1 in 11 adults, approximately 463 million people have diabetes mellitus worldwide. In Sri Lanka, 1 in 5 adults has either diabetes or pre-diabetes and one third of those with diabetes are undiagnosed.

Type 2 DM which is characterized by hyperglycemia is the most frequent type of diabetes, accounting for around $90 \%$ of all diabetic cases. Recent clinical findings explore that the underlying pathways in the pathogenesis of diabetic complications are mainly due to oxidative stress generated by over production of reactive oxygen species (ROS) during hyperglycemia. Therefore, diabetes mellitus is recognized as a redox disease. Uncontrolled hyperglycemia causes tissue damage through several biochemical mechanisms thereby leading to a variety of diabetic associated complications including nephropathy, neuropathy, peripheral vascular diseases, and retinopathy. In this review, we focus on possible mechanisms of tissue damage due to oxidative stress in type 2 DM patients during hyperglycemia. Furthermore, we discuss some of the major complications of post type 2 DM such as cardiovascular disease (CVD) and nephropathy. Thereby, in this review an attempt has been made to sum up the available literature regarding post diabetic complications and possible mechanisms enabling the researchers to conduct innovative research in this area.
\end{abstract}

Keywords: Diabetes Mellitus; Oxidative stress; ROS; Hyperglycemia, Tissue damage 


\section{Introduction}

Diabetes is considered as a serious chronic condition which is among the top ten causes of death in adults. It causes a major influence on the lives and also on the well-being of the individuals globally [1]. The number of diabetes adults in the world has increased up to 422 million in 2014 due to, population growth and ageing [2]. In 2017, the number was 451 million and it anticipated that individuals with diabetes would increase up to 693 million by 2045 [3]. In the last three decades the number of diabetes mellitus cases has quadrupled and it has become the seventh leading cause of death in 2017. Type 2 diabetes mellitus (T2DM) was the sixth major cause of disability in 2015 [2, 4, 5]. T2DM considered as a metabolic disorder while it is characterized by hyperglycemia. It can occur due to reduced insulin secretion, insulin resistance or due to a combination of both reasons $[6,7]$. Insulin insensitivity, obesity and decline of pancreas $\beta$ - cell dysfunction is related with hyperglycemia [8]. Hyperglycemia is the condition of presence of high glucose concentrations in blood due to failure in insulin stimulated glucose uptake by muscles [9]. About 1 in every 11 adults worldwide have diabetes mellitus and $90 \%$ of these adults have type 2 diabetes mellitus [10]. Biochemical factors which may increase the risk for T2D are, oxidative stress, increased generation of inflammatory cytokines, and mitochondrial dysfunction. Since glucose transporters are essential for the glucose homeostasis in the body, dysfunctional glucose transport proteins may also play a considerable role in the development hyperglycemia [11]. DM could bring up long term tissue damage, dysfunction and entire failure of many vital organs which lead to multiple organ impairment [12]. It has been suggested that hyperglycemia causes tissue damage through number of biochemical mechanisms $[13,14]$.

\section{Oxidative stress associated with hyperglycemia}

During diabetes, collapse of insulin-stimulated glucose uptake by insulin dependent muscles lead to elevated glucose concentrations in blood, which is theoretically defined as a hyperglycemic condition. Consequently, uptake of glucose by insulin-independent tissues gets triggered. Elevated influx of glucose into cells enhances both oxidant production and impairment of cellular antioxidant defenses by promoting multiple interacting biochemical mechanisms such as increased intracellular AGE formation, activated protein kinase C pathway, polyol pathway flux and increased oxidation of fatty acid $[9,14]$. Thereby the oxidative stress emerges, when the cellular oxidant production rate surpasses the rate of cellular oxidant scavenging ability $[15,16]$. There is much more evidence to state that oxidative stress is involved in the origin of diabetic complications through tissue damage [9].

\section{Possible mechanisms for tissue damage due to hyperglycemia}

It has been demonstrated that hyperglycemia causes tissue damage through number of biochemical mechanisms. Hyperglycemia increases the formation of advanced glycation end products (AGEs). Dicarbonyl AGEs such as methylglyoxal (MG), glyoxal, and 3-deoxyglucosone are formed mainly via non-enzymatic reaction of glucose and other glycating compounds derived both from glucose and from increased fatty acid oxidation with proteins. $\mathrm{MG}$ ( $\mathrm{CH} 3 \mathrm{COCHO})$ is a powerful glycating agent formed from glyceraldehyde 3phosphate (GA) and dihydroxyacetone phosphate (DHAP) in the cells [14, 17].

Furthermore, MG can be formed, in low concentrations, in the cells from proteins and lipids. MG is capable of glycating DNA, RNA and proteins. Glycated DNA, RNA and proteins cannot perform their normal biochemical function in the cells $[14,18,19]$. MG also modifies proteins, which are required in the regulation of gene transcription in endothelial cells. Furthermore, the extracellular matrix proteins which are glycated/ modified by AGE precursors interact with AGE receptors on cell surfaces and are then taken up by the cells. 
The newly entered glycated protein may activate a pleiotropic transcription factor, nuclear factor (NF)-kB, which initiates multiple pathological changes in the cells including changes in the process of gene expression [14].

Hyperglycemia increases the biosynthesis of diacylglycerol from glucose through triose phosphate. Elevated formation of reactive oxygen species during hyperglycemia suppresses the function of glycolytic enzyme Glyceraldehyde 3- phosphate dehydrogenase, GAPDH. Inhibition of GAPDH causes the raising of intracellular levels of diacylglycerol precursor, triose phosphate. Thereby increases the synthesis of DAG via triose phosphate. Elevated levels of DAG could greatly enhance the activity of Protein Kinase C (PKC) system. Protein Kinase Cs are considered as a family of at least eleven isoforms which regulates cellular functions by phosphorylating various target proteins. PKCs plays a significant role in signal transduction and cell regulation. Literature suggests that possible reactions between advanced glycation end products and relevant cell surface receptors could also enhance the functional ability of PKC isoforms. Ultimately persistent and excessive activation of several PKC isoforms in patients with T2DM causes biochemical anomalies and tissue injuries engendered by diabetes induced ROS [14, 19].

Hyperglycemia and insulin resistant leads to elevate the fatty acid oxidation and it increases the flux of fructose 6-phospahate into hexosamine pathway. In this pathway fructose 6-phosphate is synthesized and converted into glucosamine 6-phosphate. It catalyzed by the glutamine-fructose6-phosphate amidotransferase (GFAT). The products of gluosamine 6-phosphate inhibits the activity of endothelial nitric oxide synthase (eNOS). Therefore, increased flux of fructose6-phosphate leads to functional impairments in the vasculature $[8,14,20]$.

Hyperglycemia also impacts on the polyol pathway flux. Due to increased concentration of intracellular glucose some of them are converted into polyalcohol sorbitol by the enzyme called aldose reductase. In this reaction nicotinamide adenine dinucleotide phosphate (NADPH $+\mathrm{H}+$ ) is oxidized to NADP+ and is reduced back to NADPH $+\mathrm{H}+$ by the enzyme called glutathione reductase (GR). In this reaction reduced glutathione (GSH) is oxidized to GSH disulfide (GS-GS). Thus, hyperglycemia increases the consumption of GSH, decreases the concentration of intracellular GSH and renders the cell sensitive to oxidative stress [8, 14, 20].

Hyperglycemia increases the production of free radical superoxide in the mitochondria. Free radicals in mitochondria oxidize DNA, RNA, proteins, amino acids, and vitamins. The oxidized biochemical species increases oxidative stress which cause cell injuries [8, 14, 19]. Therefore, some of the biochemicals in the blood that are considered as markers of oxidative stress are elevated in blood form T2DM patients. Oxidative stress is increased due to high amount of free radical formation and decreased bioavailability of antioxidants in the cells or in the blood. Thus, hyperglycemia in T2DM patients causes biochemical changes in the body, which increases the risk for disease [14, 21].

\section{Diabetes Mellitus related CVD and aminothiol status}

Cardiovascular diseases (CVD) resulting from atherosclerosis have been identified as a main reason of mortality and morbidity in diabetes mellitus patients including type 2 diabetes mellitus; further type 2 diabetes mellitus is characterized by an increased risk for the development of micro and macro -vascular complications $[22,23,24,25]$.

Scientific literature supports the notion that oxidative stress related mechanisms have a significant involvement in the development of cardiovascular complications in type 2 diabetes mellitus patients [22, 26]. 
Studies conducted to determine their clinical relevance have been focused on several plasma markers including Homocysteine (Hcy), Cysteine (Cys), Glutathione (GSH) and Cysteinylglycine (CysGly), Malonaldehyde (MDA) and total antioxidant status (TAS). Homocysteine and cysteine are two of the main contributors in the pathogenesis of atherosclerosis and the process of increasing the risk of CVD during T2DM. Cysteinylglycine which is resulting from the degradation of GSH catalyzed by $\gamma$-glutamylcysteine transferase is recognized as a powerful oxidant. Malonaldehyde is considered as a marker of lipid peroxidation and thereby considered as a marker for oxidative stress. Certain research findings have elaborated that concentrations of MDA and Hcy in plasma are considerably higher and GSH levels are considerably lower in female type 2 diabetic patients when compared to controls. In male patients, only Hcy levels have depicted a significant elevation when compared to controls group. And a significant correlation has been observed between elevated serum glucose concentration and increased GGT activity in diabetic patients. Further, significant positive correlations have been discovered between Cys and Hcy and between GSH and Hcy in both controls and patients. Scientists have concluded GSH and MDA levels as clinical indicators of oxidative stress associated with type 2 diabetic mellitus, mostly in female patients [22].

\section{Conclusion}

In this review, we discussed DM related hyperglycemia and how it causes tissue damage through several biochemical mechanisms. All these biochemical mechanisms are directly linked with oxidative stress which arises as a result of prolong hyperglycemia. We suggest, in future, diabetic related complications should be addressed by focusing on oxidative stress and, accountable specific biomarkers must be identified to ensure early diagnosis of diabetic related complications. Thereby, adverse health impacts suffered by diabetic mellitus patients would be diminished through early medical interventions. So, we encourage future researchers to come up with more and more clinical trials and investigations to explore more related biomarkers.

\section{Acknowledgements}

First and foremost, we would like to express our heartiest gratitude to Department of Natural sciences, Faculty of Engineering and science, University of Agder, Kristiansand, Norway and then General Sir John Kotelawala Defence University, Sri Lanka for the support provided in all aspects. 


\section{References}

1. Saeedi, P., Petersohn, I., Salpea, P., Malanda, B., Karuranga, S., Unwin, N., Colagiuri, S., Guariguata, L., Motala, A.A., Ogurtsova, K., Shaw, J.E., 2019. Global and regional diabetes prevalence estimates for 2019 and projections for 2030 and 2045 : Results from the International Diabetes Federation Diabetes Atlas, Diabetes research and clinical practice 157, p. 107843.

2. Ashcroft, F.M., Rorsman, P., 2012. Diabetes mellitus and the $\beta$ cell: the last ten years, Cell 148(6), pp. $1160-1171$.

3. Cho, N., Shaw, J.E., Karuranga, S., Huang, Y., da Rocha Fernandes, J.D., Ohlrogge, A.W., Malanda, B., 2018. IDF Diabetes Atlas: Global estimates of diabetes prevalence for 2017 and projections for 2045, Diabetes research and clinical practice 138, pp. $271-281$.

4. Ackon, E.B., Francis, A.Y., Eliezer, T., Efui, A.A.M., Sampson, D., Oppong, A.B., 2019. Waist circumference and hip circumference as potential predictors of visceral fat estimate among type 2 diabetic patients at the Komfo Anokye Teaching Hospital (KATH), KumasiGhana, Alexandria Journal of Medicine 55(1), pp. 49-56.

5. Roglic, G., Varghese, C., Cowan, M., 2016. Global Report on Diabetes (World Health Organization). In WHO Library Cataloguing-inPublication Data. WHO.

6. Tiwari, B.K., Pandey, K.B., Abidi, A.B., Rizvi, S.I., 2013. Markers of oxidative stress during diabetes mellitus, Journal of biomarkers, 2013

7. DeFronzo, R.A., Ferrannini, E., Groop, L., Henry, R.R., Herman, W.H., Holst, J.J., Hu, F.B., Kahn, C.R., Raz, I., Shulman, G.I.,Simonson, D.C., 2015. Type 2 diabetes mellitus, Nature reviews Disease primers 1(1), pp. 1-22.

8. Stumvoll, M., Goldstein, B.J., Van Haeften, T.W., 2005. Type 2 diabetes: principles of pathogenesis and therapy, The Lancet 365(9467), pp. 1333-1346.

9. King, G.L., Loeken, M.R., 2004. Hyperglycemia-induced oxidative stress in diabetic complications, Histochemistry and cell biology 122(4), pp. 333-338.

10Zheng, Y., Ley, S.H., Hu, F.B., 2018. Global aetiology and epidemiology of type 2 diabetes mellitus and its complications, Nature Reviews Endocrinology 14(2), p. 88.

11 Shepherd, P.R., Kahn, B.B., 1999. Glucose transporters and insulin action-implications for insulin resistance and diabetes mellitus, New England Journal of Medicine 341(4), pp. 248-257.

12 American Diabetes A. Diagnosis and classification of diabetes mellitus, Diabetes care. 2009;32 Suppl 1(Suppl 1):S62-S7.

13 Kawahito, S., Kitahata, H., Oshita, S., 2009. Problems associated with glucose toxicity: role of hyperglycemia-induced oxidative stress, World journal of gastroenterology: WJG 15(33), p. 4137.

14 Giacco, F., Brownlee, M., 2010. Oxidative stress and diabetic complications, Circulation research 107(9), pp. 1058-70.

15 Bonnefont-Rousselot, D., 2004. The role of antioxidant micronutrients in the prevention of diabetic complications, Treatments in endocrinology 3(1), pp. 41-52.

16Bikkad, M.D., Somwanshi, S.D., Ghuge, S.H., Nagane, N., 2014. Oxidative Stress in Type II Diabetes Mellitus.

17Sato, T., Iwaki, M., Shimogaito, N., Wu, X., Yamagishi, S-i., Takeuchi, M., 2006. TAGE (toxic AGEs) theory in diabetic complications, Current Molecular Medicine 6(3), pp. 351-8. 
18Thornalley, P.J., 1996. formation, modification of proteins and nucleic acids, and enzymatic detoxification-a role in pathogenesis and antiproliferative chemotherapy, Gen Pharmacol 27, pp. 565-73.

19 Aronson, D., Rayfield, E.J., 2002. How hyperglycemia promotes atherosclerosis: molecular mechanisms, Cardiovascular diabetology 1(1), pp. 1-10.

20 Yaribeygi, H., Atkin, S.L., Sahebkar, A., 2019. A review of the molecular mechanisms of hyperglycemia-induced free radical generation leading to oxidative stress, Journal of cellular physiology 234(2), pp. 1300-12.

21 De Chiara, B., Sedda, V., Parolini, M., Campolo, J., De Maria, R., Caruso, R., Pizzi, G., Disoteo, O., Dellanoce, C., Corno, A.R., Cighetti, G., 2012. Plasma total cysteine and cardiovascular risk burden: action and interaction, The Scientific World Journal, p. 2012.

22Duman, B.S., Öztürk, M., Yilmazer, S., Hatemi, H., 2003. Thiols, malonaldehyde and total antioxidant status in the Turkish patients with type 2 diabetes mellitus, The Tohoku journal of experimental medicine 201(3), pp. 147-55.

23 Stephens, J.W., Khanolkar, M.P., Bain, S.C., 2009. The biological relevance and measurement of plasma markers of oxidative stress in diabetes and cardiovascular disease, Atherosclerosis 202(2), pp. 321-9.

24 Kannel, W.B., McGee, D.L., 1979. Diabetes and cardiovascular disease: the Framingham study, Jama 1241(19), pp. 2035-8.

25 Laakso, M., 1999. Hyperglycemia and cardiovascular disease in type 2 diabetes, Diabetes 48(5), pp. 937-42.

26Oguntibeju, O.O., 2019. Type 2 diabetes mellitus, oxidative stress and inflammation: examining the links, International journal of physiology, pathophysiology and pharmacology 11(3), p. 45 